\title{
Impact of Splenectomy on Thrombocytopenia, Chemotherapy, and Survival in Patients with Unresectable Pancreatic Cancer
}

\author{
Timothy R. Donahue • Kevork K. Kazanjian • \\ William H. Isacoff • Howard A. Reber • O. Joe Hines
}

Received: 28 December 2009 / Accepted: 23 February 2010/Published online: 23 March 2010

(C) 2010 The Author(s). This article is published with open access at Springerlink.com

\begin{abstract}
Background Patients with unresectable pancreatic cancer (PDAC) or endocrine tumors (PET) often develop splenic vein thrombosis, hypersplenism, and thrombocytopenia which limits the administration of chemotherapy.

Methods From 2001 to 2009, 15 patients with recurrent or unresectable PDAC or PET underwent splenectomy for hypersplenism and thrombocytopenia. The clinical variables of this group of patients were analyzed. The overall survival of patients with PDAC was compared to historical controls.

Results Of the 15 total patients, 13 (87\%) had PDAC and 2 (13\%) had PET. All tumors were either locally advanced $(n=6$, $40 \%)$ or metastatic $(n=9,60 \%)$. The platelet counts significantly increased after splenectomy $(p<0.01)$. All patients were able to resume chemotherapy within a median of 11.5 days (range 6-27). The patients with PDAC had a median survival of 20 months (range 4-67) from the time of diagnosis and 10.6 months (range 0.6-39.8) from the time of splenectomy.

Conclusions Splenectomy for patients with unresectable PDAC or PET who developed hypersplenism and thrombocytopenia that limited the administration of chemotherapy, significantly increased platelet counts, and led to resumption of treatment in all patients. Patients with PDAC had better disease-specific survival as compared to historical controls.
\end{abstract}

Keywords Pancreatic cancer Palliative splenectomy Pancreatic endocrine tumors

Presented at the Western Surgical Association Annual Meeting on November 11th, 2009 in San Antonio, TX.

T. R. Donahue $(\bowtie) \cdot$ K. K. Kazanjian $\cdot$ H. A. Reber $\cdot$ O. J. Hines

Department of Surgery, Division of General Surgery,

David Geffen School of Medicine at University

of California Los Angeles (UCLA),

10833 Le Conte Avenue, CHS, Room 72-215,

Los Angeles, CA 90095-6904, USA

e-mail: tdonahue@mednet.ucla.edu

W. H. Isacoff

Department of Medicine, Division of Hematology Oncology,

David Geffen School of Medicine at University

of California Los Angeles (UCLA),

Los Angeles, CA, USA

\section{Introduction}

The pancreas has a diverse cellular heterogeneity and function, and can give rise to a number of histologically distinct malignancies. Most malignant cancers originate from the ductal epithelium or endocrine cells and include pancreatic ductal adenocarcinomas (PDAC) and malignant endocrine tumors (PETs). Each histologic type has a different molecular signature and clinical course; PDACs are associated with the worst prognosis, and PETs are usually less aggressive. ${ }^{1,2}$ Most patients with PDAC (85\%) present with locally advanced or metastatic tumors that are unresectable. Treatment with gemcitabine-based chemotherapy has been shown to significantly improve survival, albeit to only a small degree. ${ }^{3}$ In contrast, PETs usually present at an earlier stage. Chemotherapy is determined by the grade of the tumor, with high-grade tumors more likely to respond. ${ }^{4-6}$ Thus, the goal of treatment for unresectable PDAC or PET is treatment with chemotherapy.

By virtue of the anatomic location of the pancreas, locally advanced PDAC or PETs can lead to thrombosis or 
occlusion of the splenic, superior mesenteric (SMV), and/or portal (PV) vein(s) with resultant hypersplenism. As in patients with cirrhosis and portal hypertension, the enhanced splenic function often produces thrombocytopenia. In addition, cytotoxic chemotherapeutic regimens, especially gemcitabine, often induce bone marrow suppression, which results in thrombocytopenia. When this occurs, many patients must stop their treatment, since serious and potentially lethal side effects could develop.

We hypothesized that a palliative splenectomy for patients with locally advanced unresectable PDAC or PETs who developed hypersplenism and thrombocytopenia that limited the administration of chemotherapy, would extend the duration of treatment and improve disease-specific survival (DSS). To investigate our hypothesis, we analyzed our experience with 15 patients who were managed with this novel treatment strategy and compared the survival of the PDAC subgroup of patients with stage-matched historical controls.

\section{Material and Methods}

\section{Patients}

Approval from the University of California, Los Angeles Office for the Protection of Research Subjects Institutional Review Board was obtained prior to initiating this study. Using a prospectively collected pancreatic cancer database, we performed a review of all patients from 2001 to 2009 with locally advanced or metastatic fine needle aspirate or biopsy (core needle, incisional, or excisional) confirmed PDAC or PET who were unresectable and underwent a splenectomy for severe thrombocytopenia that developed during administration of chemotherapy. The pathology reports were generated by one of four gastrointestinal pathologists on faculty at UCLA. The clinical, radiographic, and histopathologic findings; treatment and perioperative variables; and DSS of these patients were examined.

Clinical variables analyzed included gender, age, and stage at the time of diagnosis, and tumor histology (PDAC and PET). Radiographic variables analyzed included location of the tumor and $\mathrm{PV} / \mathrm{SMV} /$ splenic vein status (patent vs. nonpatent) on high resolution computed tomography (CT) or magnetic resonance imaging (MRI) scans. Treatment variables analyzed included the pre- and postsplenectomy chemotherapeutic regimen administered and tumor response. Variables directly related to splenectomy that were examined included length of hospital stay, need for conversion to an open operation, white blood cell count and hemoglobin immediately after surgery (postoperative day 1), and pre- and postoperative platelet counts. Preoperative platelet counts were recorded at the last clinic visit prior to surgery. Postoperative platelet counts were recorded on the day of hospital discharge. The time to resumption of chemotherapy after splenectomy was also examined.

\section{Survival Analysis}

For survival analysis, the DSS of all patients with PDAC from the time of diagnosis or splenectomy was examined. For those patients who died, the date of death was determined from the clinic charts when available, or alternatively, the social security death index (http://ssdi. rootsweb.ancestry.com/cgi-bin/ssdi.cgi) by an exact match between the patient's name and birth date. If alive, the date of last follow-up was taken as the last time the patient was seen in clinic. The two patients with PET were not included in the survival analysis, as PET are less clinically aggressive than PDAC.

\section{Statistical Analysis}

For significance analysis, $X^{2}$ and Fisher's exact test were used as appropriate. DSS was estimated using the KaplanMeier method. All statistical analyses were performed using JMP statistical software (SAS Corporation, Cary, NC). Significance was assigned at the 0.05 level.

\section{Results}

\section{Clinical, Radiographic, and Histopathologic Findings}

From 2001 to 2009,15 patients with unresectable pancreatic cancer who developed hypersplenism and thrombocytopenia, which limited the administration of their chemotherapy, underwent a splenectomy at UCLA Medical Center. The distribution of the clinical, radiographic, and histopathologic findings for these patients is listed in composite in Table 1 and individually in Table 2. Thirteen patients $(87 \%)$ had primary disease; two patients $(13 \%)$ recurred after a Whipple operation. The median age of patients was 56 years (range 25 to 62 years). Nine patients were male $(60 \%)$ and $6(40 \%)$ were female. Most patients had PDAC $(n=13,87 \%)$, while only two patients had PET $(13 \%)$. All patients had locally advanced, stage $3(n=6$, $40 \%)$ or metastatic, stage $4(n=9,60 \%)$ disease. Nine tumors $(60 \%)$ were located in the head/uncinate process and $6(40 \%)$ were located in the body/tail. On highresolution $\mathrm{CT} / \mathrm{MRI}$, the portal or splenic veins were thrombosed in $12(80 \%)$ patients (Fig. 1); the three other patients had documented splenomegaly on CT/MRI. In fact, splenomegaly was not routinely reported in the radiology report per the usual practice of the UCLA gastrointestinal 
Table 1 Composite Patients' Clinical, Radiographic, Treatment, and Histopathologic Characteristics

\begin{tabular}{ll}
\hline Age (median years) & $56(25-62)$ \\
Gender & \\
Male & $9(60 \%)$ \\
Female & $6(40 \%)$ \\
Histopathology & \\
PDAC & $13(87 \%)$ \\
PET & $2(13 \%)$ \\
Location & \\
Head/uncinate & $9(60 \%)$ \\
Body/tail & $6(40 \%)$ \\
Vein thrombosed & $12(80 \%)$ \\
Splenectomy (procedure type) & \\
Laparoscopic & $11(73 \%)$ \\
Laparoscopic converted to open & $4(27 \%)$ \\
Hospital stay (median days) & $3(2-6)$ \\
Platelet count & \\
Preoperative (median $\left.\times 10^{3}\right)$ & $87(66-160)$ \\
Postoperative (median $\left.\times 10^{3}\right)$ & $425(229-994)^{*}$ \\
\hline
\end{tabular}

$* p<0.01$

radiologists for pancreas-protocol CT scans or MRIs. The median spleen weight was $348 \mathrm{~g}$ (range 164-525) but may be an underestimate of the actual spleen size due to morcellation prior to extraction. The spleen volumes are likewise not reported for similar reasons.

\section{Treatment and Procedure Variables}

The median time from the initial diagnosis of cancer to splenectomy was 9.8 months (0.3-58) during which all patients were administered chemotherapy. Chemotherapy was stopped due to thrombocytopenia within 2 weeks of surgery for all patients. Most patients with PDAC were administered a gemcitabine-based combination therapy $(n=9,69 \%)$ both before and after splenectomy; a 5fluorouracil (5-FU)-based combination regimen was used less frequently $(n=4,31 \%)$. All patients had at least a partial tumor response to both drug treatments; there were no complete responses.

There was minimal morbidity associated with the splenectomy. A laparoscopic splenectomy was successfully performed for $11(73 \%)$ patients, while the procedure was converted to an open operation for $4(27 \%)$ patients. Excess blood loss was the primary reason for conversion. The median hospital stay was 3 days (range 2-6) and did not differ between the laparoscopic and open groups $(p>0.05)$. Recorded immediately after surgery, the white blood cell count (median $11.05 \times 10^{3} / \mu \mathrm{L}$, range $4.26 \times 10^{3}-21 \times 10^{3}$ ) and hemoglobin (median $11.75 \mathrm{~g} / \mathrm{dL}$, range 9.2-13.3) did not reveal evidence of bone marrow suppression due to preoperative chemotherapy. At the time of splenectomy, 12 patients had National Cancer Institute (NCI)/Eastern Cooperative Oncology Group (ECOG) Grade 1 thrombocytopenia (defined by $75 \times 10^{3}-150 \times 10^{3}$ ), two patients had NCI/ ECOG Grade 2 (defined by $50 \times 10^{3}-74 \times 10^{3}$ ), and one patient had impending NCI/ECOG-defined thrombocytopenia. The platelet counts significantly responded to splenectomy in all patients, preoperative (median $87 \times 10^{3} / \mu \mathrm{L}$, range $66 \times 10^{3}-160 \times 10^{3}$ ) vs. postoperative taken immediately prior to discharge (median $425 \times 10^{3} / \mu \mathrm{L}$, range $229 \times$ $\left.10^{3}-994 \times 10^{3}\right),(p<0.01)$. All patients were able to resume full dose of the same chemotherapy regimen after splenectomy within a median of 11.5 days (range 6-27).

\section{Survival Analysis}

The median follow-up for all survivors was 35 months (range 13-63) from the time of diagnosis and 25 months (range 0.6-51) from the time of splenectomy. The 13 patients with PDAC had a median survival of 20 months (range 4-67) with a 5-year DSS of $25 \%$ from the time of diagnosis, and a median DSS of 10.6 months (range 0.639.8) from the time of splenectomy (Fig. 2). Both patients with PET had well-differentiated tumors. One patient died of disease after 107 months, and the other is still alive with disease after 60 months.

\section{Discussion}

PDAC is the fourth leading cause of cancer-related deaths in the United States, with an overall 5-year survival of $4 \%$. In 2009, 42,770 patients in the USA were diagnosed with PDAC and 35,240 died from their disease. ${ }^{7}$ The poor outcome of patients with PDAC has been attributed to the advanced stage of disease at diagnosis, the poor response to current systemic and local therapies, and the aggressive biologic nature of the disease. Resection for PDAC provides the only chance for cure, but only about $15 \%$ of patients are eligible for surgery. ${ }^{8}$ Even those patients who undergo a "curative resection" have a 5-year survival rate of $35 \%$ in the best series. ${ }^{9}$ Most patients $(85 \%)$ present with locally advanced or metastatic tumors, and they have a median survival of less than 12 or 5 months, respectively. ${ }^{7}$ Chemotherapy can significantly extend DSS and decrease disease-related morbidity. ${ }^{3}$

PETs have been studied much less frequently than PDAC primarily due to their low prevalence; only about 2,500 new PETs are diagnosed annually in the United States. ${ }^{10-12}$ PETs are categorized as functional or nonfunctional depending on whether the secreted peptide is biologically active and produces a clinical syndrome; about $50 \%$ of nonfunctional PETs secrete peptides that are 
Table 2 Individual Patient's Clinical, Radiographic, Treatment, Histopathologic, and Survival Characteristics

\begin{tabular}{|c|c|c|c|c|c|c|c|c|c|c|c|}
\hline Patient & Location & Histology & Stage & Chemo & $\begin{array}{l}\text { Preop } \\
\text { Plts } \\
\left(\times 10^{3}\right)\end{array}$ & $\begin{array}{l}\text { Postop } \\
\text { Plts } \\
\left(\times 10^{3}\right)\end{array}$ & $\begin{array}{l}\text { Time to } \\
\text { chemo } \\
\text { (days) }\end{array}$ & $\begin{array}{l}\text { Status } \\
\text { at last } \\
\text { F/U }\end{array}$ & $\begin{array}{l}\text { Diagnosis } \\
\text { to surgery } \\
\text { (months) }\end{array}$ & $\begin{array}{l}\text { Survival } \\
\text { from Dx } \\
\text { (months) }\end{array}$ & $\begin{array}{l}\text { Survival } \\
\text { from } \\
\text { surgery } \\
\text { (months) }\end{array}$ \\
\hline 1 & $\mathrm{H} / \mathrm{U}$ & PDAC & 3 & $\mathrm{CiFU} / \mathrm{LV}+\mathrm{MitoC}$ & 82 & 370 & 13 & DOD & 1.37 & 4.2 & 2.8 \\
\hline 2 & $\mathrm{H} / \mathrm{U}$ & PDAC & 3 & Gemzar & 96 & 555 & 6 & DOD & 38.3 & 48.2 & 40 \\
\hline 3 & $\mathrm{H} / \mathrm{U}$ & PDAC & 4 & GTX & 87 & 425 & 14 & DOD & 57 & 15.7 & 5.9 \\
\hline 4 & $\mathrm{H} / \mathrm{U}$ & PDAC & 3 & GTX & 86 & 321 & 14 & DOD & 57.03 & 67.2 & 9.4 \\
\hline 5 & $\mathrm{H} / \mathrm{U}$ & PDAC & 3 & GFLIP & 87 & 533 & 22 & AWD & 38.3 & 63.5 & 25.2 \\
\hline 6 & $\mathrm{~B} / \mathrm{T}$ & PDAC & 4 & GFLIP & 81 & 403 & - & AWD & 4.4 & 17.9 & 13.4 \\
\hline 7 & $\mathrm{H} / \mathrm{U}$ & PDAC & 4 & $\begin{array}{l}\mathrm{CiFU} / \mathrm{LV} / \mathrm{MitoC} / \\
\text { Persantine }\end{array}$ & 81 & 447 & 9 & DOD & 9.2 & 20.4 & 10.6 \\
\hline 8 & $\mathrm{~B} / \mathrm{T}$ & PDAC & 3 & $\begin{array}{l}\mathrm{CiFU} / \mathrm{LV} / \mathrm{MitoC} / \\
\text { Persantine }\end{array}$ & 91 & 361 & 20 & DOD & 9.3 & 11.2 & 1.9 \\
\hline 9 & $\mathrm{~B} / \mathrm{T}$ & $\begin{array}{l}\text { PET } \\
\text { (well-diff.) }\end{array}$ & 4 & Temodar/Xeloda & 66 & 864 & 10 & AWD & 9.6 & 60.3 & 50.7 \\
\hline 10 & $\mathrm{H} / \mathrm{U}$ & PDAC & 3 & Gemzar & 113 & 300 & - & DOD & 10.3 & 20.4 & 10.1 \\
\hline 11 & $\mathrm{~B} / \mathrm{T}$ & $\begin{array}{l}\text { PET } \\
\text { (well-diff.) }\end{array}$ & 4 & VP16/Cisplatin & 111 & 679 & 27 & DOD & 5.03 & 107 & 95.8 \\
\hline 12 & $\mathrm{H} / \mathrm{U}$ & PDAC & 3 & $\mathrm{CiFU/LV/MitoC}$ & 86 & 541 & - & DOD & 10.6 & 28.3 & 17.3 \\
\hline 13 & $\mathrm{~B} / \mathrm{T}$ & PDAC & 4 & GTX & 88 & 994 & 8 & AWD & 2.2 & 34.9 & 32.7 \\
\hline 14 & $\mathrm{H} / \mathrm{U}$ & PDAC & 4 & GTX & 73 & 237 & 6 & DOD & 9.13 & 17.4 & 17.1 \\
\hline 15 & $\mathrm{~B} / \mathrm{T}$ & PDAC & 4 & GTX & 160 & 229 & 11 & AWD & 4.05 & 13.3 & 0.67 \\
\hline
\end{tabular}

$H / U$ head or uncinate tumor, $B / T$ body or tail, $D O D$ died of disease, $A W D$ alive with disease, $C i F U$ continous infusion $5 \mathrm{FU}, L V$ leukovorin, $M i t o C$ mitomycin C, Gemzar gemcitabine, GFLIP gemcitabine+5FU+leukovorin+irinotecan+persantine, Temodar temozolomide, GTX gemcitobine+taxotere

clinically silent. ${ }^{13}$ Insulinomas are the most common type of PET, and a majority are benign. ${ }^{14}$ In contrast, approximately $60 \%$ of non-insulin-secreting PETs are malignant. ${ }^{11,15}$ Due to their less aggressive clinical behavior than PDAC and resistance to most current chemotherapeutic agents, PETs are treated aggressively with resectional therapy. However, cytotoxic chemotherapy is given to patients with unresectable PETs. Therapy is determined by the grade of the tumor. ${ }^{4-6}$ Thus, chemotherapy is the primary goal of treatment for unresectable PET or PDAC for as long as the patient can tolerate it.

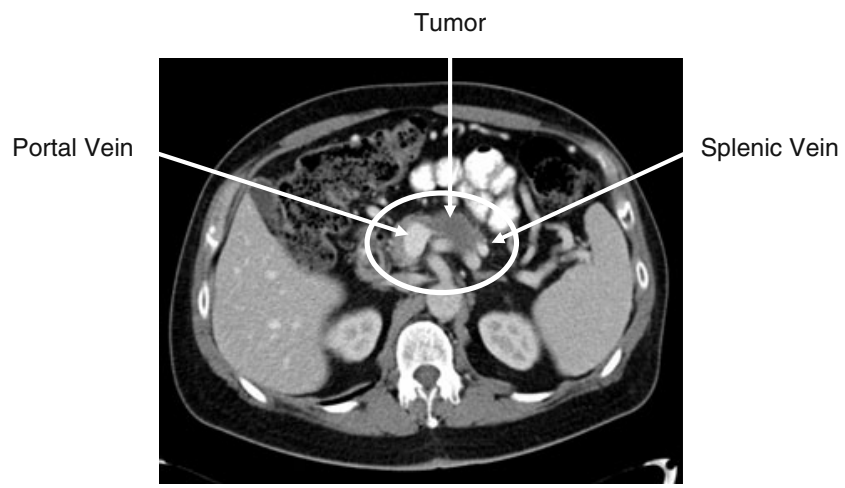

Figure 1 Representative pancreas-protocol CT scan from a patient with a PDAC located in the body/tail who has complete occlusion of the splenic vein and an enlarged spleen.
Locally advanced or recurrent pancreatic tumors of either histologic type in the head of the gland can involve the splenic vein, SMV, or PV. Tumors in the body or tail can involve the splenic vein. Either can cause venous

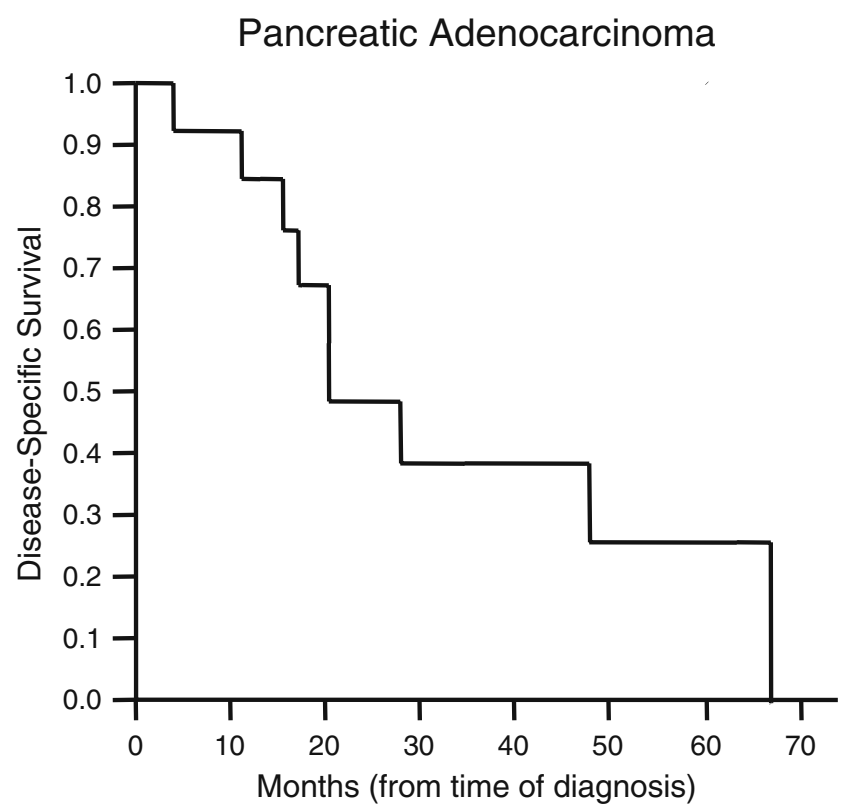

Figure 2 Disease-specific survival of 13 patients with PDAC. Median survival was 20 months (range 4-67 months). 
occlusion from compression by the tumor mass or thrombosis of the vessel. ${ }^{16}$ Left-sided portal hypertension, hypersplenism, and thrombocytopenia may result, which limits the patients' ability to tolerate aggressive chemotherapy. In this study, we examined the perioperative morbidity and effectiveness of splenectomy on restoring platelet counts to normal, administration of chemotherapy, and survival in our small series of 15 patients. A similar analysis was performed on 11 patients with hepatitis $\mathrm{C}$, cirrhosis, and portal hypertension. ${ }^{17}$ In this series, splenectomy reversed the hypersplenism-induced thrombocytopenia, and patients could resume pegylated interferon therapy.

A recent meta-analysis ${ }^{3}$ of 50 trials (7,043 participants) on the effectiveness of 5-FU- or gemcitabine-based chemotherapy and radiotherapy for inoperable pancreatic cancer found that chemotherapy can significantly improve 1 -year mortality $(p<0.00001)$ in patients with locally advanced or metastatic PDAC and can also significantly decrease morbidity. Gemcitabine-platinum combinations significantly reduced 6-month mortality on subgroup analysis $(p<0.001)$ and currently are the standard of care for the disease. Unfortunately, a number of factors often limit administration of chemotherapy to patients with pancreatic cancers. These include a poor functional or nutritional status; an unresponsive tumor and thus no clinical benefit to giving the drugs; bone marrow suppression that can result in severe anemia, leucopenia, and thrombocytopenia, ${ }^{18}$ or isolated thrombocytopenia. The potential causes of isolated thrombocytopenia include hypersplenism, bone marrow suppression with preferential inhibition of platelet production, or other very rare causes such as gemcitabine-associated thrombotic microangiopathy, ${ }^{19}$ or capecitabine (Xeloda)-induced idiopathic thrombocytopenic purpura. In fact, a major side-effect profile listed on the gemcitabine package insert includes thrombocytopenia. Thus, patients who are receiving chemotherapy, particularly gemcitabine-based regimens, are at risk of developing thrombocytopenia. With concurrent hypersplenism, the risk is even higher, as bone marrow production of platelets is usually be suppressed. Hypersplenism may unmask subclinical thrombocytopenia.

A recent study to develop a prognostic score that would predict survival after resection for PETs, using 3,851 patients from the National Cancer Data Base (19852004), found that age, grade, and distant metastases were the most significant predictors. ${ }^{20}$ Administration of adjuvant chemotherapy was not associated with increased survival. Nevertheless, cisplatin and etoposide combination therapy is effective in treating patients with poorly differentiated PETs, while streptozocin, doxorubicin, and 5-fluorouracil is the standard cytotoxic regimen for functional PETs. ${ }^{21}$ In fact, several studies suggest that PETs are more responsive to chemotherapy than endocrine tumors in other parts of the gastrointestinal tract, most notably carcinoid tumors. Our two patients with PETs who underwent splenectomy and aggressive chemotherapy have had excellent survival outcomes. As listed in Table 2, one patient is still alive with disease after 60 months and recently underwent an extensive resection of the primary tumor and multiple liver metastases. The other patient eventually died of disease after a rather long 9-year course.

Patients, with either PDAC or PET, who are offered splenectomy must demonstrate a good functional status, preferably with thrombocytopenia as the only factor limiting treatment. A complete blood cell count should be obtained preoperatively to exclude cytotoxic chemotherapy-induced bone marrow suppression as the primary cause of thrombocytopenia. If other blood elements are also low, particularly the absolute neutrophil count, then the chemotherapy should be considered as the primary cause of thrombocytopenia and splenectomy deferred. In this case, the dose of chemotherapy should be lowered or combination changed; alternatively, one might elect to give drugs that stimulate bone marrow production, such as granulocyte colony-stimulating factor or erythropoietin. If isolated thrombocytopenia is present, with the other elements normal, and there is evidence of hypersplenism on high-resolution imaging (e.g., portal vein thrombosis or an enlarged spleen), then splenectomy should be pursued. Ideally, we prefer that patients have a good response to chemotherapy, as measured by a decrease in tumor size or extent of disease on imaging and tumor markers; although, this was not the case in the present series, as patients underwent splenectomy over a wide time range from the time of diagnosis. CA19-9 is the best serum marker of response for PDAC ${ }^{22}$ chromogranin, synaptophysin, pancreatic polypeptide, or gastrin can be used for PET. ${ }^{23}$ Patients must not have end-stage disease and severe malnutrition. We require that patients have a preoperative abdominal CT or MRI scan, which are usually being done for disease surveillance during treatment. The primary tumor is evaluated to ensure that it is not growing into the splenic hilum or to note additional features (varices, etc.) that will help in planning the procedure. In addition, the abdomen is evaluated for any signs of carcinomatosis and/or ascites. By using these stringent preoperative criteria prior to splenectomy, perioperative morbidity and mortality can be minimized, and platelet counts are likely to respond.

Patients who are not operative candidates can alternatively undergo splenic artery embolization or external beam splenic irradiation, as these two treatments can also potentially reverse hypersplenism-induced thrombocytopenia. ${ }^{24}$ Embolization should be considered as second-line treatment after splenectomy because it can be associated with significant postoperative pain and splenic abscesses. ${ }^{25}$ Furthermore, splenic irradiation is rarely performed for 
hypersplenism but can be effective for relief of pain associated with splenomegaly in patients with hematologic disorders. ${ }^{26}$ In our experience, as discussed previously, splenectomy is safe and can be performed with minimal morbidity and a short hospital stay.

There were no deaths in our series; hospital stay was short (median 3 days), and patients' platelet counts responded rapidly with quick resumption of chemotherapy (median 11.5 days). The median follow-up for all survivors was 35 months (range 13-63) from the time of diagnosis. The 13 patients with PDAC had a median survival of 20 months (range 4-67) with a 5-year DSS of $25 \%$ from the time of diagnosis, and a median survival of 10.6 months (range 0.6-39.8) from the time of splenectomy.

\section{Conclusion}

In conclusion, while the optimal treatments for patients with locally advanced or metastatic PDAC or PET are in evolution, we found that our novel strategy of splenectomy for the development of hypersplenism-induced thrombocytopenia that limited chemotherapy treatment was effective. Splenectomy was performed with minimal morbidity, and was associated with a rapid increase in platelet counts and a short time before resuming chemotherapy. In addition, patients with PDAC who underwent this novel treatment strategy had significantly improved DSS as compared to historical controls.

\section{Grant Support/Assistance None}

Open Access This article is distributed under the terms of the Creative Commons Attribution Noncommercial License which permits any noncommercial use, distribution, and reproduction in any medium, provided the original author(s) and source are credited.

\section{References}

1. Modlin IM, Oberg K, Chung DC, Jensen RT, de Herder WW, Thakker RV, Caplin M, Delle Fave G, Kaltsas GA, Krenning EP, Moss SF, Nilsson O, Rindi G, Salazar R, Ruszniewski P, Sundin A. Gastroenteropancreatic neuroendocrine tumours. Lancet Oncol. 2008;9:61-72.

2. Yeo TP, Hruban RH, Leach SD, Wilentz RE, Sohn TA, Kern SE, Iacobuzio-Donahue CA, Maitra A, Goggins M, Canto MI, Abrams RA, Laheru D, Jaffee EM, Hidalgo M, Yeo CJ. Pancreatic cancer. Curr Probl Cancer 2002;26:176-275.
3. Yip D, Karapetis C, Strickland A, Steer CB, Goldstein D. Chemotherapy and radiotherapy for inoperable advanced pancreatic cancer. Cochrane Database Syst Rev. 2006;3:CD002093.

4. Moertel CG, Lefkopoulo M, Lipsitz S, Hahn RG, Klaassen D. Streptozocin-doxorubicin, streptozocin-fluorouracil or chlorozotocin in the treatment of advanced islet-cell carcinoma. N Engl J Med. 1992;326:519-523.

5. Kulke MH, Stuart K, Enzinger PC, Ryan DP, Clark JW, Muzikansky A, Vincitore M, Michelini A, Fuchs CS. Phase II study of temozolomide and thalidomide in patients with metastatic neuroendocrine tumors. J Clin Oncol. 2006;24:401-406.

6. Mitry E, Baudin E, Ducreux M, Sabourin JC, Rufie P, Aparicio T, Aparicio T, Lasser P, Elias D, Duvillard P, Schlumberger M, Rougier P. Treatment of poorly differentiated neuroendocrine tumours with etoposide and cisplatin. Br J Cancer 1999;81:13511355.

7. American Cancer Society. Cancer Facts \& Figures 2009. Atlanta: American Cancer Society; 2009.

8. Warshaw AL, Fernandez-del Castillo C. Pancreatic carcinoma. N Engl J Med. 1992;326:455-465.

9. Kazanjian KK, Hines OJ, Duffy JP, Yoon DY, Cortina G, Reber HA. Improved survival following pancreaticoduodenectomy to treat adenocarcinoma of the pancreas: the influence of operative blood loss. Arch Surg. 2008;143:1166-1171.

10. Rindi G, Capella C, Solcia E. Introduction to a revised clinicopathological classification of neuroendocrine tumors of the gastroenteropancreatic tract. Q J Nucl Med. 2000;44:13-21.

11. Kazanjian KK, Reber HA, Hines OJ. Resection of pancreatic neuroendocrine tumors: results of 70 cases. Arch Surg. 2006;141:765-769; discussion 769-770.

12. Winter JM, Cameron JL, Campbell KA, Arnold MA, Chang DC, Coleman J, Hodgin MB, Sauter PK, Hruban RH, Riall TS, Schulick RD, Choti MA, Lillemoe KD, Yeo CJ. 1423 pancreaticoduodenectomies for pancreatic cancer: a single-institution experience. J Gastrointest Surg. 2006;10:1199-1210; discussion $1210-1191$.

13. Vagefi PA, Razo O, Deshpande V, McGrath DJ, Lauwers GY, Thayer SP, Warshaw AL, Fernandez-Del Castillo C. Evolving patterns in the detection and outcomes of pancreatic neuroendocrine neoplasms: the Massachusetts General Hospital experience from 1977 to 2005. Arch Surg. 2007;142:347-354.

14. Grant CS. Insulinoma. Best Pract Res Clin Gastroenterol. 2005;19:783-798.

15. Tucker ON, Crotty PL, Conlon KC. The management of insulinoma. Br J Surg. 2006;93:264-275.

16. Tseng JF, Tamm EP, Lee JE, Pisters PW, Evans DB. Venous resection in pancreatic cancer surgery. Best Pract Res Clin Gastroenterol. 2006;20:349-364.

17. Kercher KW, Carbonell AM, Heniford BT, Matthews BD, Cunningham DM, Reindollar RW. Laparoscopic splenectomy reverses thrombocytopenia in patients with hepatitis $\mathrm{C}$ cirrhosis and portal hypertension. J Gastrointest Surg. 2004;8:120-126.

18. Picozzi VJ, Kozarek RA, Traverso LW. Interferon-based adjuvant chemoradiation therapy after pancreaticoduodenectomy for pancreatic adenocarcinoma. Am J Surg. 2003;185:476480 .

19. Humphreys BD, Sharman JP, Henderson JM, Clark JW, Marks PW, Rennke HG, Zhu AX, Magee CC. Gemcitabine-associated thrombotic microangiopathy. Cancer 2004;100:2664-2670.

20. Bilimoria KY, Talamonti MS, Tomlinson JS, Stewart AK, Winchester DP, Ko CY, Bentrem DJ. Prognostic score predicting 
survival after resection of pancreatic neuroendocrine tumors: analysis of 3851 patients. Ann Surg. 2008;247:490-500.

21. Goldin SB, Aston J, Wahi MM. Sporadically occurring functional pancreatic endocrine tumors: review of recent literature. Curr Opin Oncol. 2008;20:25-33.

22. Reni M, Cereda S, Balzano G, Passoni P, Rognone A, Fugazza C, Mazza E, Zerbi A, Di Carlo V, Villa E. Carbohydrate antigen 19-9 change during chemotherapy for advanced pancreatic adenocarcinoma. Cancer, 2009;115:2630-2639.

23. Halfdanarson TR, Rubin J, Farnell MB, Grant CS, Petersen GM. Pancreatic endocrine neoplasms: epidemiology and prognosis of pancreatic endocrine tumors. Endocr Relat Cancer 2008;15:409427.

24. Wang HY, Shih SC, Lin SC, Chang WS, Wang TE, Lin FJ, Yang FS. Partial splenic embolization: 12-month hematological effects and complications. Hepatogastroenterology 2008;55:18381842 .

25. Yoshida H, Mamada Y, Taniai N, Tajiri T. Partial splenic embolization. Hepatol Res. 2008;38:225-233.

26. Jyothirmayi R, Coltart S. An audit of the indications for and techniques of palliative splenic radiotherapy in the UK. Clin Oncol (R Coll Radiol) 2005;17:192-194. 